\title{
Tozinameran (BNT162b2) Vaccine: The Journey from Preclinical Research to Clinical Trials and Authorization
}

\author{
Nimrat Khehra, ${ }^{1}$ Inderbir Padda, ${ }^{2}$ Urooj Jaferi, ${ }^{3}$ Harshan Atwal, ${ }^{1}$ Shreya Narain, ${ }^{4}$ and Mayur S. Parmar ${ }^{4,5}$ (D)
}

Received 17 February 2021; accepted 15 May 2021; published online 7 June 2021

\begin{abstract}
Vaccination development and production was an essential question for the prevention and global control of COVID-19. The strong support from governing authorities such as Operation Warp Speed and robust funding has led to the development and authorization of the tozinameran (BNT162b2) vaccine. The BNT162b2 vaccine is a lipid nanoparticleencapsulated mRNA that encodes for SARS-CoV-2 spike protein, the main site for neutralizing antibodies. Once it binds with the host cells, the lipid nanoparticles enable the transfer of the RNA, causing S antigens' expression of the SARS-CoV-2, conferring immunity. The vaccine is administered as a 2-dose regime 21 days apart for individuals 16 years and older. PfizerBioNTech's BNT162b2 vaccine was the first candidate to receive FDA-Emergency Use Authorization (EUA) on December 11, 2020. During phase 2/3 clinical trials, $95 \%$ efficacy was reported among 37,706 participants over the age of 16 who received the BNT162b2 vaccination; additionally, $52 \%$ efficacy was noted 12 days following the administration of the first dose of BNT162b2, reflecting early protection of COVID-19. The BNT162b2 vaccine has exhibited $100 \%$ efficacy in clinical trials of adolescents between the ages of 12 and 15. Clinical trials in pregnant women and children under the age of 12 are expected to also exhibit promising results. This review article encompasses tozinameran (BNT162b2) vaccine journey, summarizing the BNT162b1 and BNT162b2 vaccines from preclinical studies, clinical trial phases, dosages, immune response, adverse effects, and FDA-EUA.
\end{abstract}

KEY WORDS: COVID-19; SARS-CoV-2; Pfizer; BioNTech; vaccine; clinical trials; preclinical trials; BNT162b1; BNT162b2 ; tozinameran; efficacy; antibody response; safety; adverse effects; phase 1 trials; phase 2 trials; phase 1/2 trials; phase $2 / 3$ trials; phase 3 trials; pregnant women; adolescents; children; real-world outcomes.

\section{INTRODUCTION}

Since the rapid emergence of the RNA virus SARSCoV-2 and the first reported case in Wuhan, China, in December 2019, early efforts were made to develop the world's first COVID-19 vaccination. Consequently, a global pandemic was announced by the World Health Organization (WHO) on March 11, 2020, and has since affected over 200 countries globally: as of April 19, 2021, over 141,057,106 confirmed cases of COVID-19 and 3,015,043 deaths worldwide [1]. The unpredictable nature of SARSCoV-2 affects individuals of all age groups with the virus's

\footnotetext{
${ }^{1}$ Saint James School of Medicine, Arnos Vale, Saint Vincent and the Grenadines.

${ }^{2}$ University of Washington, Seattle, WA, USA.

${ }^{3}$ All Saints University School of Medicine, Roseau, Dominica.

${ }^{4}$ Dr. Kiran C. Patel College of Osteopathic Medicine, Nova Southeastern University, Tampa Bay Campus, Clearwater, FL, USA.

${ }^{5}$ To whom correspondence should be addressed. (e-mail: mparmar@nova.edu)
}

characteristics and COVID-19 disease manifestations not limited to pulmonary. Although pulmonary manifestations are the predominant features of the novel coronavirus, various extrapulmonary presentations such as cardiovascular, neurological, and gastrointestinal have been linked to SARS-CoV-2, leading to mortality. With the lack of definitive treatment and diverse manifestations, symptomatic management has been the center stage for treating COVID-19 patients, hence the urgency to develop this awaited vaccine. Vaccinations are fundamental for disease prevention and have historically led to the elimination and global eradication of many infectious diseases such as poliomyelitis, measles, and smallpox. The classes of vaccines currently in progress are mRNA vaccines, replicationdefective recombinant adenoviral vectors vaccines, subunit/ protein-based vaccines, DNA vaccine, and inactivated vaccines. Due to the accelerated spread of COVID-19, several developers have clinical trials with conjoined phases to expedite the vaccine's development. This review summarizes the BNT162b1 and BNT162b2 vaccines from preclinical studies, clinical trial phases, dosages, immune 
response, adverse effects, and FDA-EUA (Emergency Use Authorization).

\section{METHODOLOGY}

A review was conducted using electronic databases such as PubMed, Google Scholar, Pubmed Central, and Government databases and the most up-to-date news articles and vaccine developer press releases to obtain related research studies, published from February 2020 to April 2021. Articles were selected based on keywords "Coronavirus," "COVID19 Vaccine," "SARS-CoV-2," "Pfizer vaccine," "Vaccine," "COVID-19 vaccination trials", "Vaccine efficacy," "Vaccine safety," "Clinical trials," "FDA approval," "Preclinical trials," "Phase 1 clinical trials," "Phase 2 clinical trials," "Phase 3 clinical trials," "Phase 1/2 clinical trials," "Phase 2/3 clinical trials," "Pfizer vaccine immune response," "children," "adolescents," "pregnant women," "real world outcomes," "effectiveness," "Pfizer adverse effects." Based on the relevance of the topic, articles were selected and interpreted accordingly.

\section{REVIEW}

\section{BNT162b Vaccine}

Pfizer and, a German company, BioNTech have collaboratively developed a lipid nanoparticle (LNP) encapsulating a nucleoside-modified messenger RNA (modRNA) that encodes for the SARS-CoV-2 spike (S) protein, the main site for neutralizing antibodies [2]. The LNP guides the modRNA's transfer within the targeted host cells, further resulting in the expression of SARS-CoV-2 S antigens, providing protection and immunogenicity against COVID-19 [2].

\section{Preclinical Trials}

From the safety and immune response data collected and assessed on four BNT162 RNA candidates, BNT162b1 and BNT162b2 emerged as strong candidates [3]. BNT162b1 and BNT162b2 vaccine candidates were administered prophylactically in the preclinical trials in select animal species, such as rhesus macaques and mice [3]. All animal species that participated in the preclinical trials were not infected with COVID-19. During the trial, macaques were randomly chosen for two intramuscular (I.M.) injections of $100 \mu \mathrm{g}$ BNT162b2 or $100 \mu \mathrm{g}$ saline [3]. The first injection was given on day 1 , and the second injection was given on day 21 [3]. Macaques that received immunization with $100 \mu \mathrm{g}$ of BNT162b2 showed favorable $\mathrm{CD}^{+}$and $\mathrm{CD}^{+}$responses, high levels of neutralizing antibodies, and no presence of viral SARS-CoV-2 RNA or lung infection in the lower respiratory tract, as early as 3 days after immunization [3]. On the other hand, macaques that received saline injections showed very high viral loads of SARS-CoV-2 [3].

Additionally, the preclinical trials studied prophylactic immunization of BNT162b2 in murine models, specifically in $\mathrm{BALB} / \mathrm{c}$ mice [3]. A single intramuscular dose of $0.2,1$, or $5 \mu \mathrm{g}$ BNT162b2 was randomly administered [3]. A robust T cell $\mathrm{CD}^{+}, \mathrm{IFN} \gamma^{+}, \mathrm{CD} 8^{+}$immune response, and pseudovirus neutralizing antibodies were observed up to day 28 in the murine models [3]. Due to BNT162b1 and BNT162b2 vaccine candidates' efficacy and safe response in preclinical trials, the company proceeded to clinical trials [3]. Phase 1 and phase 2 were combined for the first part of the trials [3].

\section{Preclinical Trials: Safety and Efficacy}

The preclinical trials additionally compared the efficacy and safety of BNT162b2 in animal models at different doses [3]. During the trial, macaques were randomly administered two I.M. injections of $30 \mu \mathrm{g}$ or $100 \mu \mathrm{g}$ BNT162b2 or saline at days 0 and 21 [3]. After both doses were given, neutralizing antibody titers were studied and showed a significant increase in the $30 \mu \mathrm{g}$ group and $100 \mu \mathrm{g}$ group at days 35 and 28 , respectively [3]. Both doses effectively produced a TH1 immune response with high IFN- $\gamma$, IL-2, and TNF- $\alpha$ levels, indicating a robust $\mathrm{CD}^{+}$and $\mathrm{CD} 8^{+} \mathrm{T}$ cell response in terms of the vaccines' safety and antiviral properties [3]. The preclinical trials further supported the multinational placebo-controlled, observer-blinded clinical trials.

\section{Phase 1/2 Clinical Trials}

In Germany, the study design in phase $1 / 2$ took place between April 23, 2020, and May 22, 2020, consisting of 60 healthy male and nonpregnant female participants $(96.7 \%$ Caucasian, one African American, and one Asian) between the ages of 18 and 55 years divided into $1 \mu \mathrm{g}, 10 \mu \mathrm{g}, 30 \mu \mathrm{g}$, and $50 \mu \mathrm{g}$ dose groups. There were initially twelve participants per dose group, and these participants were injected intramuscularly on day 1 and day 22 [2]. Due to unforeseen circumstances unrelated to the phase $1 / 2$ clinical trials, one participant from the $10 \mu \mathrm{g}$ dose group, and one participant from the $50 \mu \mathrm{g}$ dose group withdrew from the trial [2]. Another set of twelve participants were a part of the $60 \mu \mathrm{g}$ dose group received only a single vaccination dose on day 1 compared to the $1 \mu \mathrm{g}, 10 \mu \mathrm{g}, 30 \mu \mathrm{g}$, and $50 \mu \mathrm{g}$ participants receiving a dual-dose regimen [2]. On day 1 , mild to moderate adverse effects were noted in the $10 \mu \mathrm{g}$ and $30 \mu \mathrm{g}$ dose groups, as expected [2]. Within 7 days of each vaccination, symptoms such as fever, chills, headache, joint pain, muscle pain, and pain at the injection site were common findings [2]. Pain and tenderness localized to the injection site were common complaints in both initial (day 1) and booster (day 22) immunizations [2]. There were no serious adverse effects in any of the dose groups; thus, none of the participants withdrew from the study due to serious adverse effects [2].

\section{Phase 1/2 Clinical Trials: Antibody Response}

\section{BNT162b1}

Participants immunized with BNT162b1 expressed a robust dose-dependent antibody response [2]. The geometric mean concentrations (GMC) of the receptor-binding domain (RBD) IgG were assessed 21 days after the first immunization, in $1 \mu \mathrm{g}, 10 \mu \mathrm{g}, 30 \mu \mathrm{g}$, and $50 \mu \mathrm{g}$ dose groups, with the concentrations ranging from 265 to $1672 \mathrm{U} / \mathrm{ml}$ [2]. One week after the booster dose (day 29), the geometric mean concentrations ranged from 2015 to $25,006 \mathrm{U} / \mathrm{ml}$ [2]. However, 21 days following the booster dose, on day 43 of phase 
$1 / 2$ clinical trials, the RBD-binding IgG GMC ranged from 3920 to $18,289 \mathrm{U} / \mathrm{ml}$ [2]. In the $60 \mu \mathrm{g}$ dose group, the RBDbinding $\mathrm{IgG}$ GMC was $1058 \mathrm{U} / \mathrm{ml}$ on day 29 following immunization, suggesting that a dual-dose regimen may be required to increase antibody titers [2].

Following the administration of BNT162b1, neutralizing antibody titers were also assessed in participants of phase $1 / 2$ trials [2]. Twenty-one days following the initial immunization, geometric mean titers (GMT) were increased accordingly depending on the dose group [2]. Seven days following the second immunization (day 29), the neutralizing geometric mean titers markedly increased with the GMT of 36 in the $1 \mu \mathrm{g}$ dose group, 158 in the $10 \mu \mathrm{g}$ dose group, 308 in the $30 \mu \mathrm{g}$ dose group, and 578 in the 50 ug dose group [2]. Unfortunately, the neutralizing titers decreased when assessed on day 43 of the clinical trial, following the second immunization [2].

During the phase $1 / 2$ clinical trials, $\mathrm{CD}^{+}$and $\mathrm{CD}^{+} \mathrm{T}$ cells were evaluated following the first and second administration of the BNT162b1 vaccine using an ex vivo IFN-gamma ELISpot with peripheral blood mononuclear cells (PBMCs) [2]; 36/48 participants from the $1 \mu \mathrm{g}$ to the $50 \mu \mathrm{g}$ dose groups were assessed before their first vaccination (day 1 ) and 7 days following their second immunization (day 29); $94.4 \%(34 / 36)$ of the participants who were a part of the $10 \mu \mathrm{g}$ dose group or higher $(30 \mu \mathrm{g}, 50 \mu \mathrm{g})$ elicited a strong RBD-specific $\mathrm{CD}^{+}$immune response. Additionally, $80.6 \%$ (29/36) of participants elicited a robust $\mathrm{CD}^{+}$immune response [2].

\section{$B N T 162 b 2$}

During the phase $1 / 2$ clinical trials, the BNT162b2 vaccine took precedence over BNT162b1 due to higher tolerability; participants experienced mild to moderate systemic effects of fever, fatigue, and chills with indolent serious adverse effects [4]. However, the BNT162b2 GMT was notably similar to the BNT162b1 vaccine when two $30 \mu \mathrm{g}$ doses were administered 21 days apart [4]. Nonetheless, the neutralizing antibody GMT was higher in participants between the ages of 65 and 85 compared to the GMT of participants infected with COVID-19 [4]. As opposed to BNT162b1, BNT162b2 T cell responses exhibited a preferential degree of epitopes exclusive to SARS-CoV-2 antigen as well as greater $\mathrm{CD}^{+}$and $\mathrm{CD} 8^{+} \mathrm{T}$ cell responses [4]. The $\mathrm{T}$ cell responses were also noted against the receptor-binding domain (RBD), and the spike glycoprotein, exclusive to the BNT162b2 vaccine [4]. The manufacturers consider that the great extent of spike T-cell epitopes recognition may produce steady responses across various populations, including the elderly [4]. The selection of BNT162b2 to move forward to the phase $2 / 3$ clinical trial was due to its overall tolerability profile and immune response in preclinical and clinical phase trials [4].

\section{BNT162b1 vs. BNT162b2}

Pfizer and BioNTech evaluated its two major candidates, BNT162b1 and BNT162b2, to support the selection of a single vaccine candidate by examining the dose levels for safety, tolerability, and its generation of robust immune responses, and in turn, its efficacy. Similarly, BNT162b1 and
BNT162b2 share the same modRNA platform, RNA production and purification processes, and formulation of lipid nanoparticles [5]. Conversely, the BNT162b1 candidate encodes for a secreted trimerized SARS-CoV-2 receptorbinding domain, whereas the BNT162b2 encodes for a membrane-anchored SARS-CoV-2 full-length spike, stabilized in the prefusion conformation [5]. Furthermore, due to a lower percentage of incidence and severity of systemic reactions, BNT162b2 was the preferred candidate to progress to subsequent clinical trials as opposed to the BNT162b1 candidate [5]. Considering comparable antibody immune responses in both candidates, the main factor aiding the $30 \mu \mathrm{g}$ dose level of BNT162b2 vaccine candidate choice over BNT162b1 was its more moderate systemic reactogenicity profile, especially in the elderly [5, 6]. Although BNT162b1 is a strong vaccine candidate, BNT162b2 provides a favorable balance of immunogenicity and reactogenicity, especially at the $30 \mu \mathrm{g}$ dose level $[5,6]$.

\section{Phase 2/3 Clinical Trials}

From July 2020 to November 2020, the multination phase 3 randomized, placebo-controlled, observer-blinded clinical trials consisting of subjects from diverse backgrounds took place in the USA, Germany, Turkey, South Africa, Brazil, and Argentina [7,8]. Initially, 43,548 participants were randomized of which 43,448 participants received either the BNT162b2 vaccine candidate or placebo [7]. The procedures consisted of random assignment in a 1:1 ratio of subjects either being vaccinated with $30 \mu \mathrm{g}$ of the BNT162b2 vaccine or receiving a saline placebo [7]; 21,720 subjects received the BNT162b2 vaccination and 21,728 received the placebo [7]. From the 43,448 participants, data were reported on 37,706 subjects [7]. All subjects received a second dosage 21 days apart [7].

\section{Phase 2/3 Clinical Trials: Efficacy}

There is a $95 \%$ efficacy of the BNT162b2 vaccine with a $95 \%$ confidence interval ranging from 90.3 to 97.6 among the 43,448 participants who received the BNT162b2 vaccination [7]. Vaccine efficacy was noted to fare well among all subgroups such as age, sex, race-ethnicity, and obesity in elderly and young age groups [7]. Additionally, the efficacy of the BNT162b2 vaccine was assessed between doses indicating $52 \%$ efficacy with a $95 \%$ confidence interval ranging from 29.5 to 68.4 , reflecting early protection of COVID-19 12 days following administration of the first dose of the vaccine (39 BNT162b2 group and 82 placebo group) [7].

\section{Phase 2/3 Clinical Trials: Adverse Effects}

A healthcare professional, a nurse researcher, had published an article sharing her experience as a participant during the BNT162b2 vaccine clinical trial [9]. She was subject to random assignment to receive either the vaccine candidate or the placebo [9]. The first dosage was administered to her without any discerning effects other than the arm's soreness, and she was scheduled to receive the second part of the vaccination 1 month later [9]. Following the second dose administration, the participant experienced 
injection site pain more rapidly than the first dosage [9]. She reported light-headedness, chills, nausea, and headache towards the night [9]. Her symptoms progressively worsened during her sleep, accompanying a fever of $99.4^{\circ} \mathrm{F}\left(37.4^{\circ} \mathrm{C}\right)$ [9]. The fever progressively worsened during her sleep, reporting at $104.9^{\circ} \mathrm{F}\left(40.5^{\circ} \mathrm{C}\right)$, prompting supportive management with acetaminophen. Her symptoms had resolved gradually over the day, with the injection site tenderness outlasting [9]. The adverse effects experienced following the second dosage were anticipated as it was commonly reported during the phase 1 clinical trial of the vaccine [9].

\section{Phase 2/3 Clinical Trials: Safety}

Following the administration of the dual-dose vaccine in phase 3 clinical trials, safety was examined 7 days after each dose; participants noted local and systemic adverse effects and the use of antipyretic medications via solicited electronic diaries [7]. In addition, unprompted adverse effects were assessed 1 month following the second dose of the vaccine, while unprompted serious adverse effects were evaluated 6 months following the second dose [7]. Seven days following the administration of the last dose of the vaccine, mild discomfort near the injection site was the most common adverse effect noted that was present for 1-2 days; $1 \%$ of the participants spanning all age groups within this clinical trial exhibited severe localized pain [7]. Moreover, the younger population of participants reported considerable pain at the injection site as compared to the elderly population $(>55$ years) [7]. However, localized pain at the injection site did not differ from the first and second doses of the vaccine [7]. Importantly, participants within this clinical trial did not experience grade 4 local reactions [7].

Systemic effects were observed in the population group between the ages of 16 and 55; in contrast, the elderly participants ( $>55$ years) experienced the least number of systemic effects as opposed to the younger participants [7]. The most common systemic effects among the younger subset of participants were fatigue and headache; $59 \%$ of the participants reported fatigue, while $52 \%$ reported headache following the administration of the second dose of the vaccine. Also, among the elderly population group, 51\% reported fatigue, and $39 \%$ reported headache [7].

Fever was also one of the commonly experienced systemic effects following the second dose; $16 \%$ of the younger participants between the ages of 16 and 55 reported fever, whereas $11 \%$ of the elderly population over the age of 55 experienced systemic effects likewise. Younger participants used antipyretic or pain medications as compared to the elderly subset. Fever in both age groups occurred 1-2 days following the second dose of vaccination but subsided relatively fast [7].

\section{Phase 2/3 Clinical Trials: Approval}

The UK was the first country to grant government approval for the emergency use of the BNT162b2 vaccine candidate on December 2, 2020, by the Medicines and Healthcare products Regulatory Agency (MHRA) [10, 11]. The first dosage was administered successfully shortly then after. Canada was the second country to authorize emergency approval following section 5 of the interim order for the BNT162b2 vaccine candidate from governing authorities on December 9, 2020, 7 days after the approval from the UK [12, 13]. Canada is working closely with Canada's Public Health Agency and its international regulatory counterparts for the vaccine's pharmacovigilance and post-market safety [13].

Soon after approval from the UK and Canada, the USFDA concluded their meeting authorizing emergency use approval (EUA) of the BNT162b2 vaccine on December 11, 2020, supported by the Secretary of Health and Human Services (HHS) [14, 15]. The emergency use is authorized for individuals 16 years of age and older. EUA is issued by the FDA when no other alternatives are feasible [15]. Europe is the latest to receive authorization from the European Commission for the BNT162b2 vaccine for its use in 27 European Union (EU) member states [16].

\section{Phase 3 Clinical Trials: Adolescents}

In February 2021, phase 3 clinical trials were conducted in 2260 young participants in the USA between the ages of 12 and 15 , reflecting $100 \%$ efficacy post-BNT162b2 vaccination (30 $\mu \mathrm{g}$ dose) $[17,18]$. The placebo group consisted of 1129 participants, of which 18 cases were SARS-CoV-2 positive compared to 0 infected participants from the 1131 participants in the vaccinated group [17]. The antibody responses elicited in this age group were significantly greater than the 16-25 age group [17]. One month following the second dose administration, the neutralizing antibody geometric mean titers were 1239.5, indicating the generation of a robust immune response compared to the 16-25 age group with neutralizing antibody geometric titers of 705.1 [17]. Adverse effects noted in the 12-15 age group were relatively similar to the 16-25 age group [17]. Due to these findings, Emergency Use Authorization and EU Conditional Marketing Authorization of BNT162b2 in adolescents are highly expected to take place following approval from the US Food and Drug Administration (FDA) and the European Medicines Agency (EMA) [17].

\section{Phase 2/3 Clinical Trials: Pregnant Women}

In February 2021, phase 2/3 clinical trials in the USA, Canada, the UK, Brazil, Chile, South Africa, Mozambique, and Spain consisting of approximately 4000 pregnant women, over the age of 18 , between the gestation periods of 24 and 34 weeks, are partaking in this trial for a duration of 7-10 months [19]. Imperatively, pregnant women constitute a vulnerable population, thus the necessitation of its separate trial [19].

\section{Phase 1/2/3 Clinical Trials: Children Under 12 Years Old}

In March 2021, phase 1/2/3 clinical trials took place in the USA and Europe to select the desired dose and assess the safety, immune responses, and efficacy following the administration of the BNT162b2 vaccine in approximately 4644 children between the ages of 6 months and 11 years [19]. The trial included a dual-dose regimen administered 3 weeks (21 days) apart; the participants in this study were divided into 
three age groups: 6 months to 2 years, 2-5 years old, and 5-11 years of age [19].

In phase 1 of this seamless phase $1 / 2 / 3$ clinical trial, the appropriate dose conferring robust immune responses is the primary target [19]. Each of the three age groups will be administered 3 different dosages $(10 \mu \mathrm{g}, 20 \mu \mathrm{g}, 30 \mu \mathrm{g})$ of the BNT162b2 vaccine, in turn, assessing and selecting the preferred dose for subsequent phase $2 / 3$ trials in children under the age of $12[19,20]$ (Table I).

\section{BNT162b2 Vaccine: Real-World Outcomes}

\section{Overall Effectiveness}

Pfizer and BioNTech commenced the global distribution of the acclaimed BNT162b2 vaccine in December 2020 [21]. The response pertaining to the effectiveness of this vaccine has been profoundly positive [21].

Elderly ( $>70$ years) that were administered the first dose of the BNT162b2 vaccine reflected a significant reduction in the number of COVID-19-induced deaths by $85 \%$ [22]. Significant protection against symptomatic COVID-19 following the first dose of the vaccine (peaked immunity between 28 and 34 days postvaccination) by $61 \%$ with a $95 \%$ confidence interval of 51 to $69 \%$; however, immunity plateaued shortly thereafter [22]. Immunity following the dual-dose regimen markedly increased to $85-90 \%$ [22].

Elderly ( $>80$ years) who received one dose of the BNT162b2 vaccine yet experienced COVID-19 symptoms, thereafter, had supplementary immunity by $43 \%$ with a $95 \%$ confidence interval ranging from 33 to $52 \%$, markedly reducing emergency hospitalization rates [22]. The number of deaths in this age group substantially decreased due to this additional immunity by $37 \%$ with a $95 \%$ confidence interval ranging from 37 to $62 \%$ [22].

As per the Israel Ministry of Health (MoH), COVID-19 disease incidence rates have lowered significantly in individuals that were administered the dual-dose regimen of the
BNT162b2 vaccine, further confirming promising clinical trial results [21]. Additional imperative findings indicate that this vaccine has the propensity to hinder asymptomatic SARS$\mathrm{CoV}-2$ infections by $94 \%$ [21]. Data findings, from the $\mathrm{MoH}$, indicate a robust immune response approximately 14 days following the second dose of the dual-dose regimen, dramatically reducing the number of SARS-CoV-2 infections, COVID-19 cases, hospitalization rates, disease severity, progression, and mortality by $97 \%$, reflecting the effectiveness of the BNT162b2 vaccine [21].

Vaccine response and effectiveness in patients with underlying chronic diseases have been open-ended as COVID-19 disease course and complications in this population are high-risk factors for severity [23]. A study conducted by Jahn et al. [23] reported the BNT162b2 vaccination response in 72 hemodialysis patients (HDP) that were divided into four age groups: 37-59 years, 60-69 years, 70-79 years, and $80-90$ years. Vaccination response was assessed 14 days following the second $30 \mu \mathrm{g}$ dosage and subsequently compared with the control group of 16 healthy healthcare workers (HCWs). The 72 HDP $(100 \%)$ tested negative prior to receiving the first vaccination of the two-dose series. These subjects had a median age of 68 years and a median hemodialysis time of 52 months [23]. The 67/72 HDP (93\%) conferred IgG immunity 14 days following the second $30 \mu \mathrm{g}$ dose [23]. Among the $72 \mathrm{HDP}$, age had an inverse correlation with the observed antibody titers, as increased age was associated with markedly diminutive antibody titers [23]. SARS-CoV-2 infections were not reported 13 weeks postvaccination with the first dosage among the 72 HDP [23]. Compared with the control group, the $16 \mathrm{HCWs}$ had a median age of 45.5, and IgG immunity was conferred in all 16 subjects $(100 \%)$ following the second $30 \mu \mathrm{g}$ dosage of the BNT162b2 vaccine [23]. Conclusively, the antibody titers between study and control groups did not remarkably differ; in the HDP groups, median ages were the 45.5- and 54.0-yearolds and 45.5-year age groups in HCWs [23]. Conversely, sero-responses were significantly lower in the higher age

Table I. The BNT162b1 and BNT162b2 clinical trial phases, number of participants, gender distribution, age of participants, and overall findings

\begin{tabular}{|c|c|c|c|c|c|c|}
\hline Phases & Type of Trial & $\begin{array}{l}\text { Number of } \\
\text { participants }\end{array}$ & Gender & $\begin{array}{l}\text { Age of } \\
\text { Participants } \\
\text { no. (age group) }\end{array}$ & $\begin{array}{l}\text { Placebo / } \\
\text { Vaccination }\end{array}$ & Findings \\
\hline $\begin{array}{l}\text { Phase } 1 / 2 \\
(\mathrm{BNT} 162 \mathrm{~b} 1)\end{array}$ & $\begin{array}{l}\text { Placebo-controlled } \\
\text { observer-blinded } \\
\text { dose-escalationtrial }\end{array}$ & 105 & $\begin{array}{l}47 \text { male; } \\
58 \text { female }\end{array}$ & $\begin{array}{l}60(18-55 \mathrm{yr}) \\
\quad 45(65-85 \mathrm{yr})\end{array}$ & $\begin{array}{l}21 \text { Placebo; } \\
84 \text { Vaccination }\end{array}$ & $\begin{array}{l}\text { Similar serologic immune } \\
\text { response to BNT162b2 } \\
\text { The highest neutralization titers } \\
\text { were measured in samples } \\
\text { obtained on day } 28 \text { (i.e., } 7 \text { days } \\
\text { after the second dose) or on day } \\
35 \text { (i.e., } 14 \text { days after the second } \\
\text { dose). [5] }\end{array}$ \\
\hline $\begin{array}{l}\text { Phase 1/2 } \\
(\text { BNT162b2) }\end{array}$ & $\begin{array}{l}\text { Placebo-controlled } \\
\text { observer-blinded } \\
\text { dose-escalationtrial }\end{array}$ & 90 & $\begin{array}{l}36 \text { male; } \\
54 \text { female }\end{array}$ & $\begin{array}{l}45(18-55 \mathrm{yr}) ; \\
\quad 45(65-85 \mathrm{yr})\end{array}$ & $\begin{array}{l}18 \text { Placebo; } \\
72 \text { Vaccination }\end{array}$ & $\begin{array}{l}\text { BNT162b2 elicited a } \\
\text { profound favorable } \\
\text { balance of } \\
\text { immunogenicity and } \\
\text { reactogenicity at } 30 \mu \mathrm{g} \\
\text { dose level. [5] }\end{array}$ \\
\hline $\begin{array}{l}\text { Phase } 2 / 3 \\
(\text { BNT162b2) }\end{array}$ & $\begin{array}{l}\text { Multinational } \\
\text { Placebo-controlled } \\
\text { observer -blinded } \\
\text { Pivotal efficacytrial }\end{array}$ & $\begin{array}{l}43,548 \text { randomized } \\
\text { ( } 43,448 \text { received } \\
\text { injections }) \\
(37,706 \text { reported })\end{array}$ & $\begin{array}{l}\text { 19,075 male; } \\
18,631 \text { female }\end{array}$ & $\begin{array}{r}21,785(16-55 \mathrm{yr}) \\
15,921(>55 \mathrm{yr})\end{array}$ & $\begin{array}{l}\text { 118,846 Placebo; } \\
\text { 18,860 Vaccination }\end{array}$ & $\begin{array}{l}\text { Vaccine efficacy } \\
\quad \text { (Overall) - 95.0\% } \\
\text { (95\% CI, 90.0-97.6) } \\
{[15]}\end{array}$ \\
\hline
\end{tabular}


group, especially among the 60-69 and 70-79 HDP age groups [23].

\section{Effectiveness in Previously Infected SARS-CoV-2 Individuals}

As recent data emerges, the response of the BNT162b2 mRNA vaccine in previously infected individuals compared to infection-naive individuals varies to a degree $[24,25]$. Individuals that were previously infected with COVID-19 displayed a robust immune response with the presence of SARS-CoV-2-specific antibodies after only one dose of the vaccine [24, 25], whereas COVID-19 infection-naive individuals showed similar levels of SARS$\mathrm{CoV}-2$-specific antibodies to previously infected individuals after the second dose of the vaccine [24, 25]. This occurrence is possibly due to previously infected individuals having acquired natural immunity and the first vaccination acting as a recall shot for their immune system $[24,25]$.

A cohort led by Ebinger et al. [26] observed 1090 participants that received the BNT162b2 mRNA vaccine. Antibody levels were measured before or up to 3 days after the first dose, within 7-12 days after the first dose, and finally within 7-12 days after receiving the second dose [26]. The study recorded levels of spike glycoprotein-specific $\mathrm{IgG}$, specifically IgG S-receptor-binding domain (RBD) for showing the antibody response of either prior infection or vaccine and the nucleoprotein-specific $\operatorname{IgG}$ or $\operatorname{IgG}(\mathrm{N})$ for showing the antibody response to prior infection only [26]. Individuals with a prior COVID-19 infection $(n=35)$ showed higher levels of antibody after a single dose compared to infectionnaive patients $(n=225)$ after the second dose of vaccine [26]. Similar results were observed when measuring the angiotensin-converting enzyme 2 (ACE2) binding inhibition assay with higher ACE levels for previously infected individuals compared to infection-naive individuals [26]. Additionally, similar ACE levels were observed in previously infected individuals after one dose compared to infection-naive individuals' ACE levels after the second dose [26]. The study concluded that a single dose of the BNT162b2 mRNA vaccine is sufficient enough for previously infected individuals both due to the results of ACE2 binding inhibition assay and production of a strong anti-S antibody response and a complete vaccination schedule for infection-naive individuals [26].

\section{Single Dose Effects}

Since Pfizer commenced the global distribution of the BNT162b2 vaccine in December 2020, the Joint Committee on Vaccination and Immunisation (JCVI) and Public Health England (PHE) in the UK. was the first to delay the second dose to the maximum interval of 12 weeks [27]. This delayed dose allowed for research to be conducted on the effectiveness of a single dose of the BNT162b2 vaccine. In a study conducted by Jones et al. [28], healthcare workers that were vaccinated between 18 and 31 January 2021 were analyzed to determine the effectiveness of a single dosage of the BNT162b2 vaccine. Tests were conducted on individuals that reported well to work; 26 tests from unvaccinated healthcare workers were positive (26/3252), compared to 13 (13/3535) from healthcare workers $<12$ days postvaccination and 4 (4/1989) tests from healthcare workers $>12$ days postvaccination [28]. The data shows a significant decrease in the risk of asymptomatic SARS-CoV-2 infection distinctly among healthcare workers $>12$ days postvaccination [28].

Furthermore, in a study conducted by Chodick et al. [29], data was collected and analyzed of 503,875 individuals. Out of which 351,897 (69.8\%) had 13-24 days of follow-up. The collective incidence of infection was $0.57 \%$ between days 1 and 12 and $0 \cdot 27 \%$ in days 13-24 [29]. A thorough analysis of the data completed by Hunter et al. [30] indicates that the effectiveness of the BNT162b2 vaccine progressed gradually from day 14 until attaining efficacy of $90 \%$ on day 21 before a second dose. Although a single dose may be effective within 21 days, a second dose within the maximum interval of 12 weeks is still highly recommended and necessary to provide more durable/enduring protection [30].

\section{Delayed Second Dose}

The UK was the first to utilize delayed dosing of the second dosage of the two-dose COVID-19 vaccination series determined by the Joint Committee on Vaccination and Immunisation (JCVI) and Public Health England (PHE) [27]. The decision came based on their retrospective analysis of the vaccination group vs. control group from a 6-day window (15-21 days) of COVID-19 subjects. Their retrospective analysis resulted in an $89 \%$ (95\% CI, 52-97) efficacy supporting their delayed second dose decision. JVCI further concluded the study supporting $89 \%$ effectiveness continuing day 21 to day 85 without the second dose of the two-dose series [31]. Based on scientific literature, the BNT162b2 vaccination reported $52 \cdot 4 \%$ efficacy on day 22 , and $50-60 \%$ efficacy was summarized in Israel's observational cohort studies during a similar time frame [31]. The suboptimal and partial immunity conferred by a single dosage and delayed the second dosage can put individuals and populations at risk from SARS-CoV-2 and its newly emerging variant strains [31]. The BNT162b2 two-dose vaccination series administered 21 days (3 weeks) apart have reported a 94\% efficacy and should be maintained and supported to confer optimal immunity [31].

\section{Effectiveness Against SARS-CoV-2 Variants}

There have been several reports of SARS-CoV-2 variants that have emerged and have been spreading worldwide. The major variants of concern that have been discovered are the UK variant (also known as 20I/501Y.V1, VOC 202012/01, or B.1.1.7), the South Africa variant (also known as 20H/ 501Y.V2 or B.1.351), and the Brazil variant (also known as P.1) $[32,33]$. Recent reports of individuals infected with these variants have not shown a significant increase in mortality; however, a faster and more efficient spread of the variants has been observed $[32,33]$. More importantly, the variants have shown more than 10 amino acid mutations in the SARS-CoV2 spike (S) protein, which has been an area of concern for the effectiveness of the BNT162b2 vaccine against these variants $[32,33]$. 
A study by Muik et al [34] compared the efficacy of the BNT162b2 vaccine on 40 participants with the Wuhan reference strain or the B.1.1.7 variant. Serum was obtained from the 40 participants on day 7 and day 21 after both doses of the vaccine were administered and showed overall preservation of neutralizing antibodies against the B.1.1.7 variant in most cases [34]. Patients over the age of 55 showed a slight reduction in the preservation of neutralizing antibodies but were overall effective to protect against the variant [34]. The authors of the study stated that the UK B.1.1.7 variant is unlikely to evade the BNT162b2-elicited protection [34].

Similarly, the effectiveness of the BNT162b2 vaccine against several other variant strains has shown efficacy in recent studies. A clinical trial conducted by Liu et al [35] compared the efficacy of the BNT162b2 vaccine against the SARS-CoV-2 variant, USA-WA1/2020; twenty serum samples were extracted, on day 14 and day 28, from 15 participants following vaccination. Additionally, the trial engineered five recombinant variant strains, which either extracted the spike (S) protein or substituted amino acid of the receptor-binding site from variants B.1.1.7, B.1.351, P.1, and two subset mutations of B.1.351, into the USAWA1/2020 strain [35]. All of the serum samples with the USA-WA1/2020 and the variant strains showed effective neutralization of the virus after BNT162b2 vaccination [35]. The USA-WA1/2020 strain, the UK B.1.351 variant, and the Brazil P.1 variant all showed a robust neutralization of the virus at similar titers [35]. The South African variant, B.1.351, showed lower neutralization titers than the other variants; additionally, neutralization titers were also lower than the two subset mutations of B.1.351 [35]. BNT162b2-mediated protection was observed with $\mathrm{CD} 8^{+}$ $\mathrm{T}$ cell responses and high neutralization titers in the USAWA1/2020 reference strain and the variants; however, more research is needed to examine the full effect of the BNT162b2 vaccine on these specific variants [35].

\section{Adverse Effects}

Although uncommon following vaccination, severe adverse reactions and 21 cases of anaphylaxis were reported by the Centers for Disease Control and Prevention (CDC) to the Vaccine Adverse Event Reporting System (VAERS) following the administration of the first dose of BNT162b2 vaccine [36]; 1,893,360 first doses of BNT162b2 were administered between December 14 and December 23, 2020 [36]; 1,177,527 first doses were administered in women, 648,327 in men, while 67,506 did not report their sex [36]. Also, 4 of the 21 patients (19\%) were hospitalized; 3 of these 4 patients were admitted to the intensive care unit [36]. Seventeen $(81 \%)$ of the 21 recipients were treated accordingly in the emergency department [36]. Twenty (95\%) patients were discharged following appropriate management [36]. Notably, no deaths due to anaphylaxis were reported [36].

In the USA, cutaneous adverse reactions were noted in 414 individuals following the administration of an mRNA vaccine from December 2020 to February 2021 [37].
Seventy-one $(17 \%)$ of the 414 individuals who experienced cutaneous manifestations postvaccination were administered the BNT162b2 vaccine [37]. Manifestations ranged from local pain at the injection sites, urticaria, morbilliform rash, to delayed local reactions noted after the first and second doses [37]. Other rare dermatologic/cutaneous adverse reactions following mRNA vaccination included pernio/ chilblains, reactivation of herpes simplex virus, varicellazoster virus, and reactions similar to pityriasis rosea [37]. Swelling in regions where cosmetic fillers were previously administered was also noted [37]. The cutaneous similarities denoted in SARS-CoV-2 positive individuals and post mRNA vaccinations reflect the vaccine's ability to imitate the virus [37].

\section{LIMITATIONS}

This article focuses on identifying and understanding the preclinical trial, clinical trial phases, dosages, immune response, adverse effects, and FDA-EUA of the BNT162b2 vaccine. This article poses limitations as only the UK, Canada, USA, and 27 EU member states have recently granted emergency use approval, and its longterm effects are yet to be reported. The BNT162b2 vaccine clinical trials in children under the age of 12 and pregnant women are ongoing. Additional real-world studies and the latest data analyses are beneficial to further support existing findings.

\section{CONCLUSION}

This review article summarizes the journey of BNT162b1 and BNT162b2 vaccine from the preclinical phase to EUA while understanding the immune response, safety profile, dosages, and adverse effects in individuals (males and nonpregnant females) 16 years of age and older, as well as understanding additional ongoing clinical trials in pregnant women over the age of 18 and clinical trials in children under the age of 12 by the Pfizer and BioNTech collaboration. The candidate is an LNP encapsulated mRNA that encodes for the SARS-COV-2 spike protein. The vaccine candidate, tozinameran, is the first to be granted FDA-EUA, alongside the UK, Canada, and 27 EU member states. Four BNT162b candidates were initially studied, of which BNT162b1 and BNT162b2 had progressed to phase 1/ 2 while BNT162b2 proceeded to phase $2 / 3$ and received FDA-EUA at the $30 \mu \mathrm{g}$ dose level due to its favorable balance of immunogenicity and reactogenicity. Operation Warp Speed has been of tremendous aid in the manufacturing and development of the BNT162b2 vaccine without cutting corners to jeopardize its safety and efficacy. This vaccine is vital to control the further spread and stop COVID-19 globally. Alongside vaccinations, global public health measures are demanded and encouraged to prevent the spread of SARS-CoV-2. Precautionary measures such as physical distancing, quarantine, face mask use, and handwashing are fundamental public health stratagems to prevent the spread of COVID-19 within communities globally. 


\section{AUTHOR CONTRIBUTION}

I.P.: drafting of the review article, editing, interpretation of data, and revision. N.K.: drafting of the review article, editing, interpretation of data, and revision. U.J.: drafting the review article and interpretation of data. H.A.: drafting the review article and interpretation of data. S.N.: drafting the review article and interpretation of data. M.P.: conceptualization, review, editing, and supervision of review article writing process.

\section{DECLARATIONS}

Conflict of Interest The authors declare no competing interests.

\section{REFERENCES}

1. WHO. Coronavirus disease COVID-19. WHO coronavirus disease (COVID-19) dashboard. World Health Organization. April 9, 2021, [Accessed April 19, 2021 https://covid19.who.int/]

2. Sahin U, Muik A, Derhovanessian E, et al. Concurrent human antibody and TH1 type T-cell responses 2 elicited by a COVID19 RNA vaccine. medRxiv. Published July 20, 2020. doi: https:// doi.org/10.1101/2020.07.17.20140533. [Accessed January 14, 2020, https://www.medrxiv.org/content/10.1101/ 2020.07.17.20140533v1].

3. Vogel AB, Kanevsky I, Che Y, et al. Immunogenic BNT162b vaccines protect rhesus macaques from SARS-CoV-2 [published online ahead of print, 2021 Feb 1]. Nature. 2021;10.1038/s41586021-03275-y. doi:https://doi.org/10.1038/s41586-021-03275-y [Accessed February 2, 2021 https://www.nature.com/articles/ s41586-021-03275-y]

4. Pfizer. n.d. News/Pfizer and BionTech choose lead mRNA vaccine candidate against COVID-19 and commence pivotal phase 2/3 global study. https://www.pfizer.com/news/press-release/press-release-detail/pfizer-and-biontech-choose-leadmrna-vaccine-candidate- 0

5. Walsh EE, Frenck RW Jr, Falsey AR, Kitchin N, Absalon J, Gurtman A, et al. Safety and immunogenicity of two RNAbased COVID-19 vaccine candidates. N Engl J Med. 2020;383(25):2439-50. https://doi.org/10.1056/NEJMoa2027906 [Accessed April 6, 2021 https://www.nejm.org/doi/full/10.1056/ NEJMoa2027906].

6. Padda IS, Parmar M. COVID (SARS-COV-2) Vaccine. In: StatPearls. Treasure Island (FL): StatPearls Publishing; February 16, 2021. [Accessed April 11, 2021 https:// pubmed.ncbi.nlm.nih.gov/33620862/ ]

7. Polack FP, Thomas SJ, Kitchin N, et al. Safety and efficacy of the BNT162b2 mRNA COVID-19 vaccine [published online ahead of print, 2020 December 10]. N Engl J Med. 2020;10.1056/ NEJMoa2034577. doi:https://doi.org/10.1056/NEJMoa2034577 [Accessed January 14, 2021 https://www.nejm.org/doi/full/ 10.1056/NEJMoa2034577]

8. Pfizer. Pfizer and BioNTech announce publication of results from landmark phase 3 trial of BNT162b2 COVID-19 vaccine candidate in the New England Journal of Medicine. [Accessed April7th,2021https://www.pfizer.com/news/press-release/pressrelease-detail/pfizer-and-biontech-announce-publication-resultslandmark ]

9. Choi KR. A nursing researcher's experience in a COVID-19 vaccine trial. JAMA Intern Med. Published online December 07, 2020. doi:https://doi.org/10.1001/jamainternmed.2020.7087 [Accessed December 18, 2020, https://jamanetwork.com/ journals/jamainternalmedicine/fullarticle/2773790], 157, 158
10. GOV. U.K. Regulatory approval of Pfizer/BioNTech vaccine for COVID-19. [Accessed December 18, 2020,] https://www.gov.uk/ government/publications/regulatory-approval-of-pfizerbiontech-vaccine-for-covid-19]

11. Nature. The U.K. has approved a COVID vaccine - here's what scientists now want to know. ISSN 1476-4687 Nature 588, 205-206 (2020) doi: https://doi.org/10.1038/d41586-020-03441-8 [Accessed December 18, 2020 https://www.nature.com/articles/ d41586-020-03441-8

12. Government of Canada. Pfizer-BioNTech COVID-19 vaccine: what you should know. COVID-19 vaccines [Accessed December 18, 2020,] https://www.canada.ca/en/health-canada/services/ drugs-health-products/covid19-industry/drugs-vaccines-treatments/vaccines/pfizer-biontech.html

13. Government of Canada. Pfizer-BioNTech COVID-19 vaccine (tozinameran). COVID-19 vaccines and treatments portal [Accessed December 18, 2020 ]https://covid-vaccine.canada.ca/ pfizer-biontech-covid-19-vaccine/product-details

14. CDC. Morbidity and mortality weekly report (MMWR). Weekly/December 18, 2020/69(50);1922-1924 [Accessed December 18, https://www.cdc.gov/mmwr/volumes/69/wr/ mm6950e2.htm?s_cid=mm6950e2_x]

15. FDA. Pfizer-BioNTech COVID-19 vaccine [Accessed December 18, 2020 ]https://www.fda.gov/emergency-preparedness-andresponse/coronavirus-disease-2019-covid-19/pfizer-biontechcovid-19-vaccine

16. Pfizer. News/Pfizer and BioNTech receive authorization in the European Union for COVID-19 vaccine [Accessed January 14, 2021 https://www.pfizer.com/news/press-release/press-releasedetail/pfizer-and-biontech-receive-authorization-europeanu n i o $\#$ : : t e x t $=($ N Y S E \% 3 A \% 20 P F E ) \% 20and $\% 20$ BioNTech, 16\%20years\%20of\%20age\%20and

17. Pfizer. Pfizer-BioNTech announce positive topline results of pivotal COVID-19 vaccine study in adolescents. [Accessed April 7, 2021 https://www.pfizer.com/news/press-release/pressrelease-detail/pfizer-biontech-announce-positive-topline-resultspivotal]

18. Clinicaltrials.gov. A phase 3 study to evaluate the safety, tolerability, and immunogenicity of multiple production lots and dose levels of BNT162b2 against COVID-19 in healthy participants. [Accessed April 11, 2021 https://clinicaltrials.gov/ ct2/show/NCT04713553 ]

19. Pfizer. Studies in additional populations. [April 7, $2021 \mathrm{https} / /$ www.pfizer.com/science/coronavirus/vaccine/additional-population-studies ]

20. Clinicaltrials.gov. Study to evaluate the safety, tolerability, and immunogenicity of an RNA vaccine candidate against COVID-19 in healthy children $<12$ years of age [Accessed April 11, 2021 https://clinicaltrials.gov/ct2/show/NCT04816643 ]

21. Pfizer. Real-world evidence confirms high effectiveness of Pfizer and BioNTech COVID-19 vaccine and profound public health impact of vaccination one week after pandemic declared. [Accessed April 8, 2021 https://www.pfizer.com/news/press-release/press-release-detail/real-world-evidence-confirms-high-effectiveness-pfizer]

22. Iacobucci G. COVID-19: single dose of Pfizer and Oxford vaccines cuts risk of hospital admission by $80 \%$ in over 80 s, data suggest. BMJ. 2021;372:n612 doi:10.1136/bmj.n612, Accessed 17 Apr 2021. https://doi.org/10.1136/bmj.n612.

23. Jahn M, Korth J, Dorsch O, Anastasiou OE, Sorge-Hädicke B, Tyczynski B, et al. Humoral response to SARS-CoV-2 vaccination with BNT162b2 (Pfizer-BioNTech) in patients on hemodialysis. Vaccines. 2021;9(4):360. Accessed Apr 17, 2021. https:// doi.org/10.3390/vaccines 904036 .

24. Manisty C, Otter AD, Treibel TA, et al. Antibody response to first BNT162b2 dose in previously SARS-CoV-2-infected individuals. Lancet 2021; 397 (10279): P1057-1058. DOI:https:// doi.org/10.1016/S0140-6736(21)00501-8. [Accessed April 19, 2021, https://www.thelancet.com/journals/lancet/article/ PIIS0140-6736(21)00501-8/fulltext].

25. Krammer F, Srivastava K, Alshammary H, Amoako AA, Awawda MH, Beach KF, et al. Antibody responses in seropositive persons after a single dose of SARS-CoV-2 mRNA vaccine. N Engl J Med. 2021;384:1372-4. https://doi.org/10.1056/ 
NEJMc2101667 Accessed April 19, 2021, https://www.nejm.org/ doi/full/10.1056/NEJMc2101667.

26. Ebinger JE, Fert-Bober J, Printsev I, Wu M, Sun N, Prostko JC, et al. Antibody responses to the BNT162b2 mRNA vaccine in individuals previously infected with SARS-CoV-2. Nat Med. 2021. https://doi.org/10.1038/s41591-021-01325-6 Accessed April 19, 2021, https://www.nature.com/articles/s41591-021-01325-6.

27. Gov.UK. Optimising the COVID-19 vaccination programme for maximum short-term impact. Updated January 26, 2021. Accessed 18 Apr, 2021 https://www.gov.uk/government/publications/prioritising-the-first-covid-19-vaccine-dose-jcvi-statement/ optimising-the-covid-19-vaccination-programme-for-maximumshort-term-impact]

28. Jones NK, Rivett L, Seaman S, et al. Single-dose BNT162b2 vaccine protects against asymptomatic SARS-CoV-2 infection [published online ahead of print, 2021 Apr 8]. Elife. 2021;10:e68808. Accessed Apr 11, 2021, https:// elifesciences.org/articles/68808]

29. Chodick G, Tene L, Patalon T, Gazit S, et al. The effectiveness of the first dose of BNT162b2 vaccine in reducing SARS-CoV-2 infection 13-24 days after immunization: real-world evidence. medRxiv. Preprint posted online January 29, 2021. Accessed Apr 18, 2021 https://www.medrxiv.org/content/10.1101/ 2021.01.27.21250612v1]

30. Hunter PR, Brainard J. Estimating the effectiveness of the Pfizer COVID-19 BNT162b2 vaccine after a single dose. A reanalysis of a study of 'real-world' vaccination outcomes from Israel. medRxiv. Preprint posted online February 3, 2021. Accessed Apr 18, 2021 https://www.medrxiv.org/content/ 10.1101/2021.02.01.21250957v1\#disqus_thread]

31. Robertson JFR, Sewell HF, Stewart M. Delayed second dose of the BNT162b2 vaccine: innovation or misguided conjecture? Lancet. 2021;397(10277):879-80. https://doi.org/10.1016/S01406736(21)00455-4 Accessed April 17, 2021, https:// www.thelancet.com/journals/lancet/article/PIIS01406736(21)00455-4/fulltext.

32. Xie X, Liu Y, Liu J, et al. Neutralization of SARS-CoV-2 spike 69/70 deletion, E484K and N501Y variants by BNT162b2 vaccine-elicited sera. Nat Med. 2021;27:620-1. https://doi.org/ 10.1038/s41591-021-01270-4 Accessed April 19, 2021 https:// www.nature.com/articles/s41591-021-01270-4?s=08.

33. Hall VJ, Foulkes S, Saei A, et al. Effectiveness of BNT162b2 mRNA vaccine against infection and COVID-19 vaccine coverage in healthcare workers in England, multicentre prospective cohort study (the SIREN Study). Lancet. 2021. https:// doi.org/10.2139/ssrn.3790399 Accessed April 19, 2021 https:// papers.ssrn.com/sol3/papers.cfm?abstract_id=3790399.

34. Muik A, Wallisch A, Sanger B, et al. Neutralization of SARSCoV-2 lineage B.1.1.7 pseudovirus by BNT162b2 vaccineelicited human sera. Science. 2021:371(6534):1152-3. https:// doi.org/10.1126/science.abg6105 Accessed April 19, 2021 https://science.sciencemag.org/content/371/6534/1152.abstract.

35. Liu Y, Liu J, Xia H, Zhang X, Fontes-Garfias CR, Swanson KA, et al. Neutralizing activity of BNT162b2-elicited serum. N Engl J Med. 2021;384:1466-8. https://doi.org/10.1056/NEJMc2102017 Accessed April 19, 2021, https://www.nejm.org/doi/full/10.1056/ NEJMc2102017.

36. Shimabukuro T, Nair N. Allergic reactions including anaphylaxis after receipt of the first dose of Pfizer-BioNTech COVID19 vaccine. JAMA. Published online January 21, 2021. doi:https://doi.org/10.1001/jama.2021.0600 [Accessed January 21, 2021 https://jamanetwork.com/journals/jama/fullarticle/ 2775646 ? guest Access Key=1441 a a 96-9dc6-4d50-85f88dcbd270a551\&utm_source=linkedin_company\&utm_mediu$\mathrm{m}=$ social_jama\&utm_term $=4423373747 \& u$ tm_campaign $=$ article_alert\&linkId=109655018 ]

37. McMahon DE, Amerson E, Rosenbach M, et al. Cutaneous reactions reported after Moderna and Pfizer COVID-19 vaccination: a registry-based study of 414 cases [published online ahead of print, 2021 Apr 7]. J Am Acad Dermatol. 2021;S01909622(21)00658-7. doi:https://doi.org/10.1016/j.jaad.2021.03.092 [Accessed April 11, 2021, https://pubmed.ncbi.nlm.nih.gov/ 33838206/]

Publisher's Note Springer Nature remains neutral with regard to jurisdictional claims in published maps and institutional affiliations. 